\title{
Evaluation of Fast-Ion Confinement Using a Radially Injected Neutral Beam in the LHD
}

\author{
Masaki OSAKABE, Sadayoshi MURAKAMI ${ }^{1)}$, Takafumi ITOH, Mitsutaka ISOBE, \\ Shinji KOBAYASHI ${ }^{2}$, Tokihiko TOKUZAWA, Kenichi NAGAOKA, Haruhisa NAKANO, \\ Katsuyoshi TSUMORI, Katsunori IKEDA, Yasuhiko TAKEIRI, Osamu KANEKO and LHD-group \\ National Institute for Fusion Science,322-6 Oroshi-cho, Toki 509-5292, Japan \\ ${ }^{1)}$ Department of Nuclear Engineering, Kyoto University, Kyoto 606-8501, Japan \\ ${ }^{2)}$ Department of Kyoto University, Uji 611-0011, Japan
}

(Received 18 January 2010 / Accepted 14 May 2010)

\begin{abstract}
The confinement properties of perpendicular fast ions, which have kinetic energies mostly perpendicular to magnetic field lines, are investigated in the Large Helical Device (LHD) using short pulse injections of neutral beams (NB). Fast-ion lifetimes are evaluated using the fast neutral decay times after NB turn-off, where the neutrals are measured by an array of silicon-diode fast neutral analyzers. We found that the lifetime normalized by the 90-degree pitch-angle scattering time can be used as a scale of fast-ion confinement properties in magnetically confined fusion devices. The good confinement properties of the LHD's inwardly shifted configuration were demonstrated using the evaluated normalized lifetimes.
\end{abstract}

(c) 2010 The Japan Society of Plasma Science and Nuclear Fusion Research

Keywords: fast-ion confinement, heliotron device, trapped particle, NB-blip, fast neutral measurement

DOI: $10.1585 /$ pfr.5.S2042

\section{Introduction}

Achieving good confinement properties of fast ions is one of the most important concerns in magnetically confined fusion devices, since it is necessary to sustain plasmas by fusion-born alpha particles to realize fusion reactors. In stellarator devices, where the confining magnetic field is provided only by magnetic coils, the heating efficiency of fusion-born alpha particles might be degraded, since the three-dimensional (3D) ripple components of magnetic field lines strongly affect the orbit topologies of fast ions. Therefore, investigating fast-ion confinement properties with 3D ripple components is important in helical devices. Moreover, the effect of 3D ripple components on fast-ion confinement has recently been recognized to be important in tokamak devices as well, since these components might be enhanced with the introduction of test blanket modules (TBMs) and/or resonant magnetic perturbation (RMP) coils in the International Thermonuclear Experimental Reactor (ITER) [1,2].

A radial neutral beam (NB) has been in operation on the Large Helical Device (LHD) since 2005. All the fast ions produced by the NB have kinetic energies largely perpendicular to the magnetic field lines. The confinement properties of perpendicular particles strongly depend on the ripple structures of the magnetic configuration. Therefore, the particles produced by the radial NB can be used as test particles to investigate the confinement properties of fast ions in various configurations of the LHD.

author'se-mail: osakabe.masaki@lhd.nifs.ac.jp
This paper presents the results of short-pulse NB injection (NB-blip) experiments using the radial NB. The confinement properties of perpendicular fast ions are investigated by measuring the decay time of fast neutrals after the NB turn-off. These fast neutrals are measured by a radial array of silicon diodes $[3,4]$, which have sight lines perpendicular to the magnetic field lines of the LHD. The experimental apparatus is described in section 2. The experimental results are shown and fast-ion confinement properties are discussed in section 3 , while section 4 presents the conclusion.

\section{Experimental Apparatus}

Figure 1 shows a schematic drawing of the LHD midplane and radial NB injection (NBI). The center line of the injector is set perpendicular to the axis of LHD plasmas in a cross section in which the plasmas are horizontally elongated, and is placed on the midplane of the LHD. An injection energy of $40 \mathrm{keV}$ is chosen for a beam composed of hydrogen ion species, and the total nominal injection power of the NBI is $6 \mathrm{MW}$. The NBI has four ion sources, which are aligned both horizontally and vertically. The locations of the ion sources are identified as UA, LA, UB, and LB, which indicate the upper-left, lower-left, upper-right, and lower-right locations, respectively. The axis of each ion source is tilted $2.76 \mathrm{deg}$. horizontally and $3.45 \mathrm{deg}$. vertically. Both the focal points and the pivot point are set to a point at $R=4.9 \mathrm{~m}$ on the midplane, and the distance from the ion sources to the point is $8.3 \mathrm{~m}$. A 


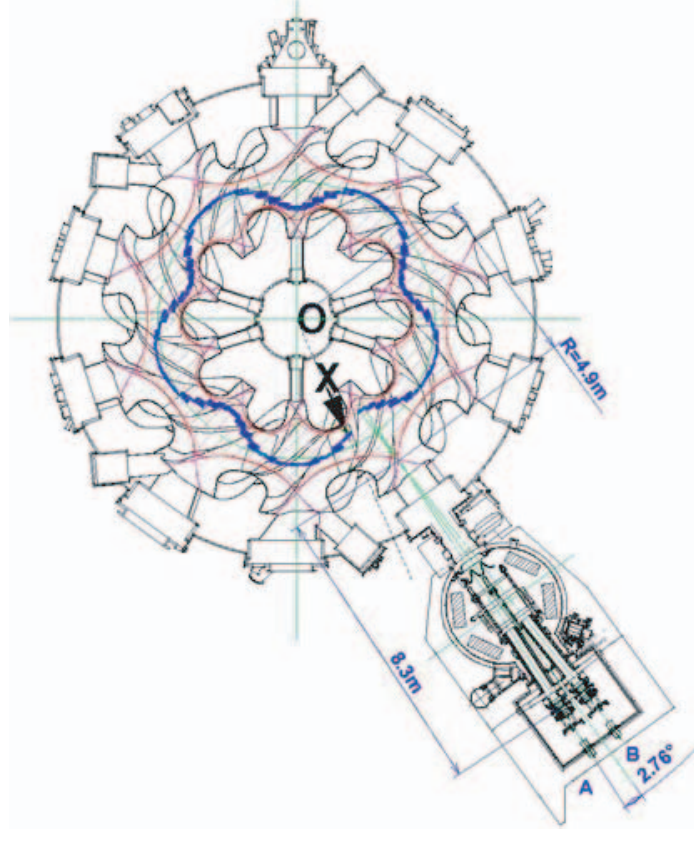

Fig. 1 Schematic drawing of cross-sectional view of the LHD midplane and radial NB injection geometry. Hatched area shows the cross section of the LHD standard configuration.
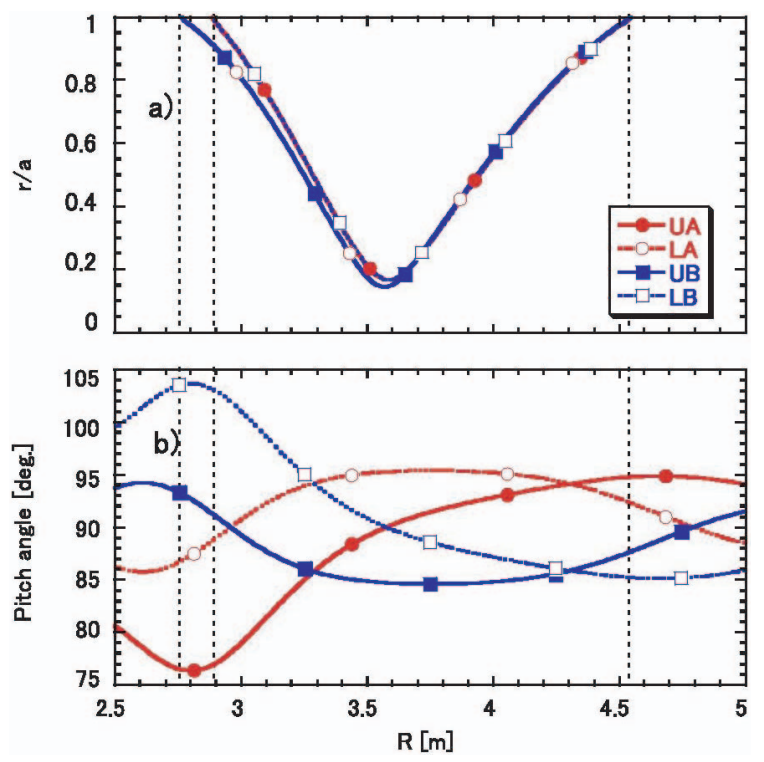

Fig. 2 (a) Normalized minor radii and (b) pitch-angle distributions along the center lines of ion sources of radial NBI in the $\sigma$-optimized $\left(R_{\mathrm{ax}}=3.6 \mathrm{~m}\right)$ configuration. Black dashed lines indicate the location of the last closed flux surface.

typical divergent angle of the beam is $1.2 \mathrm{deg}$. In Fig. 2, the normalized minor radii distribution and pitch-angle distribution along the center lines are shown for the LHD $\sigma$ optimized configuration $\left(R_{\mathrm{ax}}=3.6 \mathrm{~m}\right)$, where good particle transport is expected theoretically in stellarators [3]. $R_{\mathrm{ax}}$ denotes the major radius of the configuration's magnetic
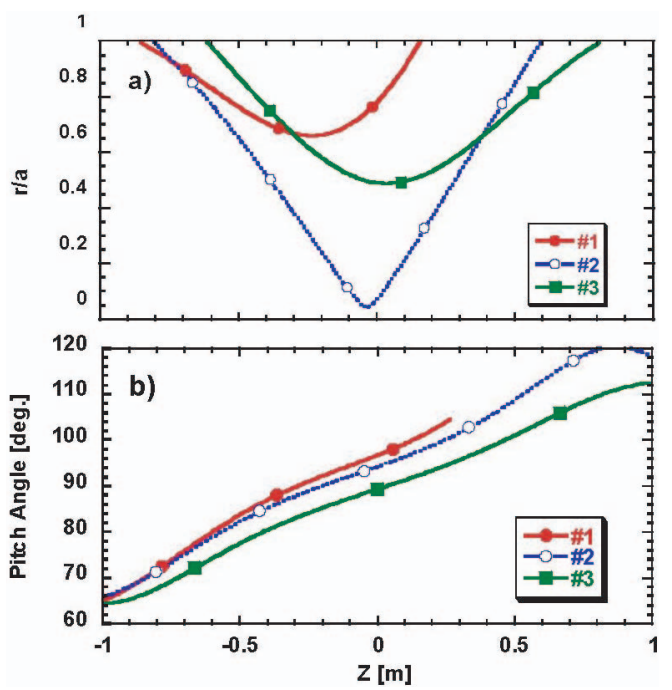

Fig. 3 (a) Normalized minor radii and (b) pitch-angle distributions along the sight lines of the Si-FNAs. Their sight

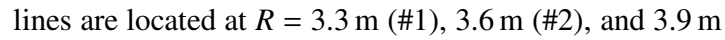
(\#3).

axis.

To investigate the confinement properties of perpendicular fast ions in the plasmas, fast neutrals produced by charge exchange between fast ions and neutrals in plasmas are measured by a fan array of fast neutral analyzers based on silicon diodes (Si-FNAs) [2,3]. The array is installed at the lower port of the LHD; its port location is indicated by an $\mathrm{X}$ in Fig. 1. Three of the Si-FNAs in the array are used for the analysis described here. Their sight lines are shown with the distributions of normalized minor radii and pitch angles in Fig. 3. The solid angles of their sight lines are $2.7 \times 10^{-5} \mathrm{sr}$ at the maximum and can be reduced to $8.1 \times 10^{-6}$ sr. In Fig. 1, a typical orbit of a deeply trapped particle, which is produced by the radial NB and can be measured by one of the Si-FNAs (\#3), is shown by a blue curve. No active sources of neutrals for charge exchange processes, i.e., pellets and/or NBs, are supplied for this diagnostic procedure. Only the neutrals penetrating from the plasma's periphery are used for this purpose. Thus, the measurement becomes line integrated along the sight lines of the FNAs. The $\mathrm{Si}$ diodes are cooled to $-15^{\circ} \mathrm{C}$ by a pair of Peltier modules to reduce thermal noise. The minimum energy ranges of the Si-FNAs are around $29 \mathrm{keV}$, and the energy resolutions are $5 \mathrm{keV}$ at full width at half maximum. Both energies are limited by the amount of thermal noise. The upper limit of the measurable energy range is $1 \mathrm{MeV}$, which is determined by the input range of the analog-todigital converters.

\section{Evaluation of Perpendicular Fast Ions with NB-blip Experiments}

The confinement properties of perpendicular fast ions are evaluated from the flux decay of fast neutrals after 

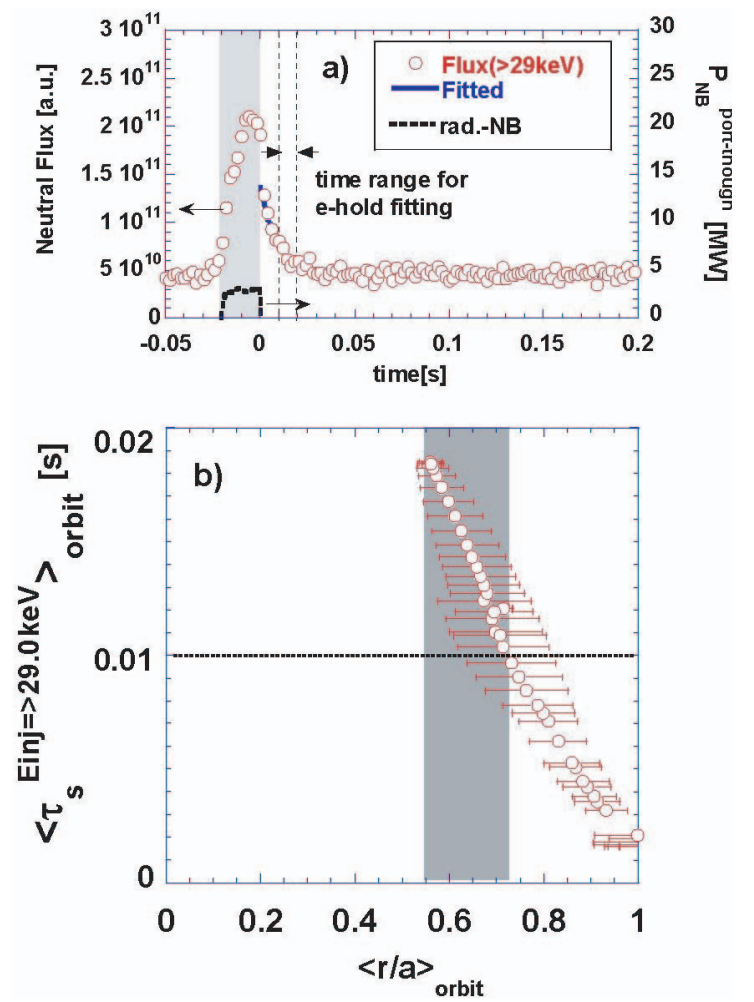

Fig. 4 (a) Typical waveform of fast neutral flux (open circles) and that of port-through power of the radial NB (dashed curves). Neutral flux measured by Si-FNA\#3 and greater than $29 \mathrm{keV}$ is shown. (b) Orbit-averaged slowing-down time distribution on the sight lines of Si-FNA\#3. Dashed lines indicate a slowing-down time of $10 \mathrm{~ms}$. Gray area indicates the region where the slowing-down time is longer than $10 \mathrm{~ms}$.

short-pulse injection of the radial NB. A typical time trace of the perpendicular fast neutrals is shown in Fig. 4(a). Signals of Si-FNA\#3 greater than $29 \mathrm{keV}$ are integrated, and those for eight NB pulses of $20 \mathrm{~ms}$ injection with plasmas at almost the same density and temperature are accumulated in the figure. Figure 4 (b) shows the slowing-down time distribution on the sight line of Si-FNA\#3. The symbol \langle\rangle$_{\text {orbit }}$ denotes an average value over the fast ion orbit, and error bars indicate the width of the orbit. For the sight line of Si-FNA\#3, the inner most observable region is $\langle r / a\rangle_{\text {orbit }}=0.55$, as shown in Fig. 3 (b). The slowingdown time from the NB injection energy, which is $36 \mathrm{keV}$ for this particular case, to $29 \mathrm{keV}$ is calculated according to the following formula and averaged along the orbits on the detector sight line:

$$
\tau_{\mathrm{s}}^{\text {Einj } \rightarrow \text { Ethres. }}=\frac{\tau_{\mathrm{se}}}{3} \ln \left(\frac{E_{\text {inj. }}^{3 / 2}+E_{\mathrm{c}}^{3 / 2}}{E_{\text {thres. }}^{3 / 2}+E_{\mathrm{c}}^{3 / 2}}\right),
$$

where $\tau_{\mathrm{se}}$ is the Spitzer slowing-down time and is calculated from the electron densities and temperatures measured by Thomson-scattering measurements and far infrared (FIR) interferometers. $E_{\mathrm{c}}$ is the critical energy at which the heating rate of fast ions to bulk electrons and that to bulk ions are equal. The neutral flux shown in Fig. 4 (a) contains spatial information on its sight line since it is a line-integrated measurement. However, if the time range of interest is specified at a certain time period after NB turn-off, the spatial region of our measurement can be localized. For example, the fast neutrals shown in Fig. 4 (a) are considered to come mostly from inside of $\langle r / a\rangle_{\text {orbit }}=$ 0.72 if we set the time range of interest to between $10 \mathrm{~ms}$ and $20 \mathrm{~ms}$ after NB turn-off. As shown in Fig. 4 (b), fast ions produced by the radial NB outside of $\langle r / a\rangle_{\text {orbit }}=0.72$ have slowing-down times shorter than $10 \mathrm{~ms}$. Thus, these particles are not measurable $10 \mathrm{~ms}$ after NB turn-off. Since the inner most region observable by Si-FNA\#3 is $\langle r / a\rangle_{\text {orbit }}$ $=0.55$, the measurement region can be localized between $\langle r / a\rangle_{\text {orbit }}=0.55$ and 0.72 in the time range between $10 \mathrm{~ms}$ and $20 \mathrm{~ms}$ after NB turn-off. In Fig. 4 (a), the decay time of the neutral flux intensities is evaluated by exponential fitting of the data points between $t=0.01$ and $0.02 \mathrm{~s}$. The result of the fitting is shown by blue lines in the figure. The evaluated decay times are compared with the averaged slowing-down time in the region between $\langle r / a\rangle_{\text {orbit }}=0.55$ and 0.72 in Fig. 5 (a). In calculating the average, the weight of $w(r / a)$ as shown below is used:

$$
w(r / a)=\frac{n_{\mathrm{e}}\left\langle\tau_{\mathrm{se}}\right\rangle_{\mathrm{orbit}} n_{0}}{\int d L\left(n_{\mathrm{e}}\left\langle\tau_{\mathrm{se}}\right\rangle_{\mathrm{orbit}} n_{0}\right)},
$$

where the integration in the denominator is done along the detector sight line. $n_{0}(r / a)$ is the neutral density calculated by the AURORA code, and the product of $n_{\mathrm{e}}(r / a)$ and $<\tau_{\mathrm{se}}>_{\text {orbit }}$ roughly represents the density profile of fast ions created by NB deposition on the sight line of the Si-FNA. Thus, the weight roughly expresses the fast neutral density profile along the sight line. The dashed line in Fig. 5 shows the line of a unity slope. The deviations of data points from this line indicate the amount of fast ion loss from the orbits on the Si-FNA sight line. In Fig. 5 (a), the deviation of data points is less significant for the $R_{\mathrm{ax}}=3.6 \mathrm{~m}$ configuration than for that of $R_{\mathrm{ax}}=3.75 \mathrm{~m}$. This indicates better confinement properties for the $R_{\mathrm{ax}}=3.6 \mathrm{~m}$ configuration and is consistent with a theoretical prediction of better confinement properties for the inwardly shifted configuration of the LHD [4]. The lifetimes of fast ions staying in their orbits can be evaluated by

$$
1 / \tau_{\text {lifetime }}=1 / \tau_{\text {decay }}-1 /\left\langle\tau_{\mathrm{s}}^{\text {Einj. } \rightarrow \text { Ethres. }}\right\rangle_{\text {orbit }} .
$$

Two candidates are responsible for the loss of fast ions from orbits on the sight lines: one is the charge exchange loss of fast ions with neutrals coming from the periphery; the other is pitch-angle scattering. The evaluated lifetimes $\left(\tau_{\text {lifetime }}\right)$ are compared with the 90 -degree pitch-angle scattering times $\left(<\tau_{\text {perp. }} .>_{\text {orbit }}\right)$ and with the characteristic times for the charge exchange loss process $\left.\left(<\tau_{\mathrm{cx}} \cdot\right\rangle_{\text {orbit }}\right)$ in Figs. 5(b) and (c), respectively. $\tau_{\text {lifetime }}$ shows a linear correlation with $\left\langle\tau_{\text {perp. }}>_{\text {orbit }}\right.$. On the other hand, the figures show an anticorrelation with $\left\langle\tau_{\mathrm{cx}} .\right\rangle_{\text {orbit }}$. Thus, the pitch-angle scattering process is considered to 

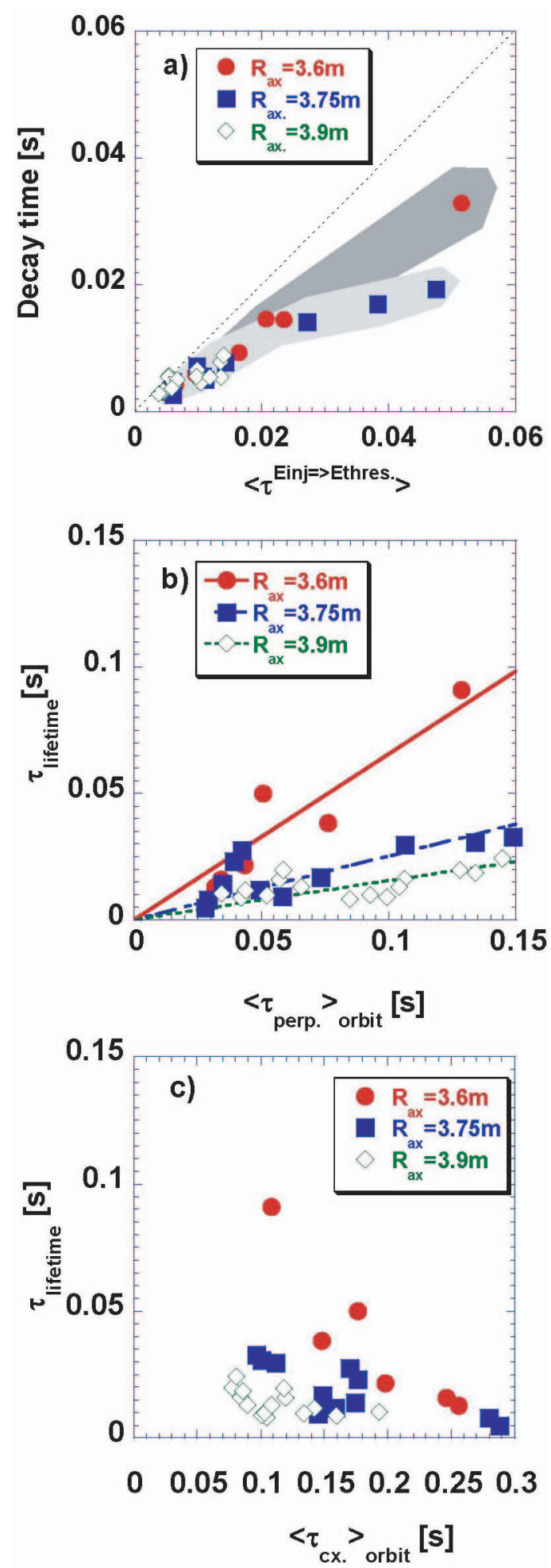

Fig. 5 Comparison of (a) orbit-averaged slowing-down times to decay times of neutral flux, (b) evaluated lifetimes to orbit-averaged 90-degree pitch-angle scattering times, and (c) charge exchange loss times. Red closed circles, blue closed squares, and green open diamonds represent the experimental results for the $R_{\mathrm{ax}}=3.6 \mathrm{~m}, 3.75 \mathrm{~m}$, and $3.9 \mathrm{~m}$ configurations, respectively, at $B_{\mathrm{t}}=2.5 \mathrm{~T}$.

be the dominant loss process of fast ions from orbits on the detector sight line. Considering the magnitudes of the solid angles of the Si-FNA sight lines, which are of the order of

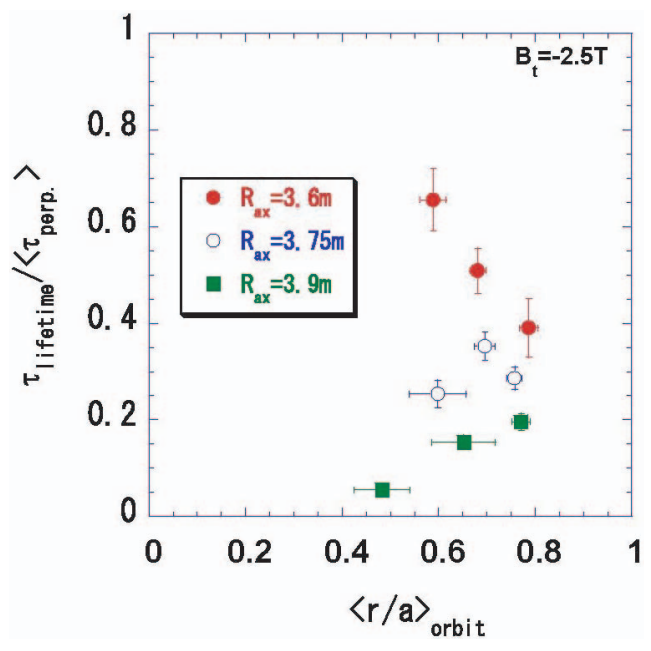

Fig. 6 Configuration dependence of normalized lifetime distributions.

$10^{-5} \mathrm{sr}$, as shown in section 2 , the lifetimes evaluated from the flux decay times are much larger than the expected loss times with a simple pitch-angle scattering process from the sight line. From the particle balance of fast ions on the $\mathrm{Si}$ FNA sight lines in velocity space, the fast-ion lifetime on the FNA sight line can be written as

$$
\frac{d N_{\mathrm{LOS}}}{d t}=-\frac{N_{\mathrm{LOS}}}{\tau_{\text {lifetime }}}=\frac{a N_{\text {outsice }}}{\tau_{\text {perp. }}}-\frac{b N_{\mathrm{LOS}}}{\tau_{\text {perp. }}},
$$

where $N_{\text {LOS }}$ and $N_{\text {outside }}$ are the number of particles inside and outside the solid angles of the sight lines in velocity space, respectively. The constants $a$ and $b$ are determined from the observation geometries. Using this equation, the lifetime normalized by the 90 -degree pitch-angle scattering time can be written as

$$
\tau_{\text {lifetime }} / \tau_{\text {perp. }}=\frac{1}{b-a \gamma}
$$

where $\gamma=N_{\text {outside }} / N_{\text {LOS. }}$. If the fast-ion confinement is good, $\gamma$ is large, since the Si-FNA sight line observes close to the birth points of perpendicular fast ions in velocity space. Therefore, the normalized lifetime, which is evaluated from the slopes of data points for each configuration in Fig. 5 (b), can be considered a good indicator of fast-ion confinement properties.

The distributions of normalized lifetimes are shown in Fig. 6. The normalized lifetimes of the inwardly shifted configuration $\left(R_{\mathrm{ax}}=3.6 \mathrm{~m}\right)$ are larger than those of the standard $\left(R_{\mathrm{ax}}=3.75 \mathrm{~m}\right)$ and outwardly shifted $\left(R_{\mathrm{ax}}=\right.$ $3.9 \mathrm{~m}$ ) configurations. This is consistent with the theoretical prediction of fast-ion confinement properties of the LHD [4]. In the outwardly shifted configuration, the normalized lifetime at the edge region is larger than that of the core region. This might be due to the particle supply in the edge region from the core by radial transport of fast ions in the poor confinement configuration. 


\section{Conclusion}

The confinement properties of perpendicular fast ions are investigated with short-pulse injection of a radial NB in the LHD. Fast-ion confinement properties of the LHD are quantified experimentally by defining the lifetimes normalized by 90 -degree pitch-angle scattering times. The evaluated lifetimes show good confinement properties of the LHD's inwardly shifted configuration, which is consistent with the theoretical predictions. A quantitative comparison with numerical simulations remains as future work.

\section{Acknowledgment}

This work is supported by the LHD project budget (NIFS09ULBB503, NIFS09ULBB512, and NIFS09KLBB301). This work is also partly supported by KAKENHI from JSPS, Nos. 18340189 and 21360457. The authors are grateful to the LHD operation group for their excellent technical support.

[1] T. Kurki-Suonio et al., Ncul. Fusion 49, 095001 (2009).

[2] Y. Liang et al., Nucl. Fusion 50, 025013 (2010).

[3] M. Osakabe et al., Proc. of the 17th International Toki Conference, Toki (2007).

[4] M. Isobe et al., Fusion Sci. Tech. 58, 426 (2010).

[5] H. E. Mynick et al., Phys. Rev. Lett. 48, 322 (1982).

[6] M. Osakabe et al., Rev. Sci. Instrum. 72, 788 (2001).

[7] S. Murakami et al., Fusion Sci. Tech. 46, 241 (2004). 